\title{
CD28-B7 blockade prevents the development of experimental autoimmune glomerulonephritis
}

\author{
John Reynolds, ${ }^{1}$ Frederick W.K. Tam, ${ }^{1}$ Anil Chandraker, ${ }^{2}$ Jennifer Smith, ${ }^{1}$ \\ Ayman M. Karkar, ${ }^{1}$ Jane Cross, ${ }^{1}$ Robert Peach, ${ }^{3}$ Mohamed H. Sayegh, ${ }^{2}$ \\ and Charles D. Pusey ${ }^{1}$
}

1Division of Medicine, Imperial College School of Medicine, Hammersmith Hospital, London W12 ONN, United Kingdom
2Brigham and Women's Hospital, Harvard Medical School, Boston, Massachusetts 02115, USA
${ }^{3}$ Bristol-Myers Squibb Co., Princeton, New Jersey 08543, USA

Address correspondence to: John Reynolds, Renal Section, Division of Medicine, Imperial College School of Medicine, Hammersmith Hospital, Du Cane Road, London W12 ONN, United Kingdom. Phone: 44-181-383-3152;

Fax: 44-181-383-2062; E-mail: john.reynolds@ic.ac.uk.

This work was presented in part at the 30th Annual Meeting of the American Society of Nephrology, November 1997. Portions of this work have appeared in abstract form (Reynolds, J., et al. 1997. J. Am. Soc. Nephrol. 8:464).

Received for publication March 4, 1999, and accepted in revised form January 25, 2000.

\begin{abstract}
Experimental autoimmune glomerulonephritis (EAG), an animal model of Goodpasture's disease, can be induced in Wistar Kyoto (WKY) rats by a single injection of rat glomerular basement membrane (GBM) in adjuvant. EAG is characterized by circulating and deposited anti-GBM antibodies, accompanied by focal necrotizing glomerulonephritis with crescent formation. The role of $T$ cells in the pathogenesis of EAG remains unclear. T-cell costimulation is provided by ligation of CD28 with either B7.1 (CD80) or B7.2 (CD86) on antigen-presenting cells, and can be inhibited by a soluble form of CTLA4 (CTLA4-Ig) that binds to both B7.1 and B7.2. We examined the effect of CD28-B7 blockade on the development of EAG using native CTLA4-Ig or mutant CTLA4-Ig (Y100F-Ig), which selectively blocks B7.1. Native CTLA4-Ig treatment ameliorated EAG by several measures, including the levels of circulating antiGBM antibodies, albuminuria, the deposition of IgG and fibrin in the glomeruli, the severity of glomerular abnormalities, and the numbers of infiltrating $T$ cells and macrophages. Y100F-Ig resulted in a similar reduction in the severity of nephritis, but produced no overall reduction in circulating anti-GBM antibodies, although there was a reduction in IgG2a antibodies. We concluded that CD28-B7 blockade reduced autoantibody production and cellular infiltration of glomeruli, and prevented target organ injury. Our results suggest a key role for B7.1 in costimulation of Th1-like autoimmune responses in the rat, and show that glomerular injury in EAG is largely dependent on cell-mediated mechanisms.
\end{abstract}

J. Clin. Invest. 105:643-651 (2000).

\section{Introduction}

Antigen-specific T-cell activation is regulated by a 2 -signal pathway. The first signal is provided by engagement of the T-cell receptor (TCR) with the antigenic peptide-MHC molecule complex on antigen-presenting cells (APC), and thus represents an antigen-specific response. However, this interaction alone is insufficient to induce optimal T-cell activation without secondary costimulatory signals; these are provided by the binding of specific receptors on $\mathrm{T}$ cells with their ligands on APC. The best-characterized and strongest costimulatory signal for interaction between $\mathrm{T}$ cells and APC is provided by CD28 and CTLA4 on T cells binding to B7.1 and B7.2 (CD80 and CD86) on APC (1-11). Costimulation via CD28 provides an important signal to antigen-stimulated $\mathrm{T}$ cells that results in enhanced activation, proliferation, and differentiation. CTLA4 is a coligand on T cells that also binds to B7.1 and B7.2 on APC, and is believed to deliver a negative signal leading to cell-cycle arrest. Because CTLA4 binds to B7 with greater affinity than does CD28, a soluble form of CTLA4 has been used to inhibit T-cell costimulation via CD28, by blocking B7.1 and B7.2 receptors on APC. Blockade of this pathway has been shown to induce specific T-cell anergy in vitro (3), and to inhibit autoimmune (12-16) and alloimmune responses in vivo. (17).

Although studies using the fusion protein CTLA4-Ig, which binds to B7.1 and B7.2, have shown suppression of cell-mediated and humoral immunity in several mouse models of autoimmune disease (12-16), it is unclear whether different costimulatory signals are delivered through CD28 depending on whether B7.1 or B7.2 is the ligand. It has been suggested that B7.1 costimulates $T$ cells for Th1 responses, and B7.2 costimulates $T$ cells for Th2 responses (8-10). CD28-B7 costimulatory blockade by CTLA4-Ig has been shown to prevent experimental autoimmune encephalomyelitis by inhibiting the production of Th 1 cytokines but sparing Th2 cytokines, thus causing a state of immune deviation toward Th2 function (14). However, recent studies using specific B7.1- and B7.2-blocking $\mathrm{mAbs}$ to prevent murine autoimmune disease produced varying data regarding to the role of these 
molecules in the immune response. In experimental autoimmune diabetes, administration of anti-B7.2 mAb ameliorated disease, whereas anti-B7.1 mAb worsened disease (15). The opposite effect was observed in experimental autoimmune encephalomyelitis, where anti-B7.1 $\mathrm{mAb}$ was effective at preventing disease, and anti-B7.2 $\mathrm{mAb}$ administration was ineffective (16). Greater understanding of the mechanisms by which costimulatory blockade works, and of the different roles of B7.1 and B7.2 in the induction of autoimmunity, is required.

Experimental autoimmune glomerulonephritis (EAG) is an experimental model of Goodpasture's disease that can be induced in genetically susceptible strains of rat by immunization with heterologous or homologous preparations of glomerular basement membrane (GBM) in adjuvant (18-21). In the model used in this study, Wistar Kyoto (WKY) rats given a single injection of collagenasesolubilized rat GBM in Freund's complete adjuvant (FCA) develop sustained anti-GBM antibody synthesis, linear deposition of IgG on the GBM, deposits of fibrin in the glomeruli, albuminuria, focal necrotizing glomerulonephritis with crescent formation, and variable lung hemorrhage (21). This model of EAG shares many characteristics with the human disease, and involves antiGBM antibodies with specificity similar to that of human autoantibodies (22). As in Goodpasture's disease, the development of nephritis is associated with both cellmediated and humoral immunity to the noncollagenous (NC1) domain of the $\alpha 3$ chain of type IV collagen, or $\alpha 3(\mathrm{IV}) \mathrm{NC} 1$. Work from other groups using related models supports the idea that the main target antigen is generally the same in EAG as in Goodpasture's disease (23-26). In addition, anti-GBM antibodies from rats and mice with EAG have been shown to be pathogenic in passive transfer experiments $(27,28)$, demonstrating a role for humoral immunity in the development of EAG.

There is also increasing evidence explaining the role of T lymphocytes in the pathogenesis of EAG $(20,28-35)$. In the model used here, EAG is characterized by an influx of $\mathrm{T}$ cells that precedes macrophage infiltration and glomerular injury (29). Transfer of CD $4^{+} \mathrm{T}$ lymphocytes purified from the spleens of rats with EAG can prime naive recipients for the disease (30); in vitro stimulation of these cells by GBM before transfer confers the ability to induce anti-GBM antibody production in recipients (Reynolds et al., unpublished observation). $\mathrm{T}$ cells from animals with EAG also proliferate in vitro in response to purified $\alpha 3$ (IV)NC1 domains of rat GBM (31). Our previous work has demonstrated that cyclosporin A (32) and anti-CD4 mAb (W3/25) (33) are effective in preventing EAG in the Brown Norway rat. We also have evidence that both anti-CD8 mAb (OX8) (34) and oral administration of GBM antigen (35) are effective in preventing the development of crescentic nephritis in the WKY rat. Thus, EAG in the WKY rat is a T cell-dependent autoimmune disease that closely resembles human Goodpasture's disease.

A soluble chimeric CTLA4 fusion protein (CTLA4-Ig), constructed by linking the variable domain of the CTLA4 gene with the $\mathrm{CH} 2$ and $\mathrm{CH} 3$ domains of the human $\mathrm{IgG} 1$ constant region (5), has been shown to be effective in reducing the severity of EAG in the WKY rat when given at the time of immunization or during the course of the disease $(36,37)$. However, the rats developed antibody to the human IgG, which was associated with a reduction in levels of circulating CTLA4-Ig. More effective treatment may therefore be achieved by using a CTLA4 construct fused with mouse $\operatorname{IgG}$, which is itself less immunogenic to the rat. To further understand the pathogenic mechanisms involved in the development of EAG, and the different roles of B7.1 and B7.2 in costimulation, we examined the in vivo effects of native CTLA4, and of a mutant form of CTLA4 (Y100F, made by substituting tyrosine for phenylalanine at position 100) that selectively blocks B7.1 (10). Murine native and mutant CTLA4 were linked to murine IgG, and human native and mutant CTLA4 were linked to human IgG. The results of this study demonstrate that CD28-B7 blockade is effective in preventing EAG, providing further evidence for the importance of $T$ cell-mediated responses in crescentic nephritis. In addition, blockade of B7.1 alone was almost as effective in preventing EAG as was blockade of both B7.1 and B7.2. We speculate that blockade of B7.1 has a selective effect on Th1-like immune responses in the rat, based on the reduction in IgG2a anti-GBM antibodies, but confirmation of this depends on phenotypic characterization of engaged lymphocytes $(14,38)$. Strategies targeting $T$ cell costimulation in a selective fashion may therefore provide a novel approach to the treatment of Goodpasture's disease.

\section{Methods}

\section{Experimental animals}

Male WKY rats, aged 8-12 weeks and weighing 120-150 g, were purchased from Charles River UK Ltd. (Margate, United Kingdom). All animals were housed in standard conditions and had free access to normal laboratory diet and water.

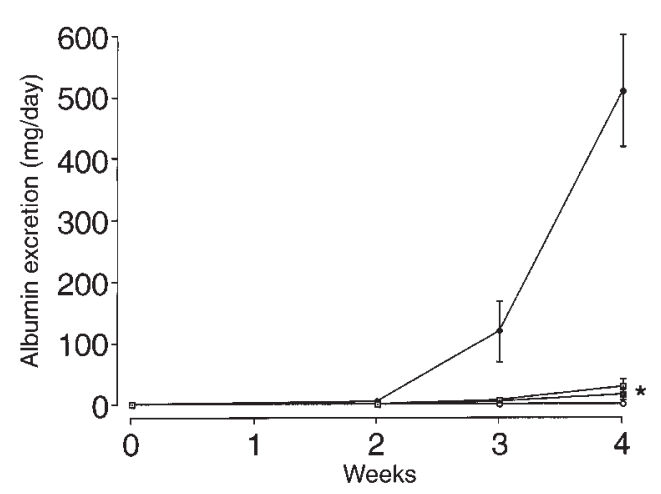

Figure 1

Effect of native CTLA4-Ig or Y100F-Ig treatment on albuminuria in groups of WKY rats $(n=5-8)$ with EAG. Results shown represent the mean \pm SD of each group: positive control (filled circles), CTLA4-Ig (filled squares), Y100F-Ig (open squares), and negative control (open circles). ( ${ }^{*} P<0.001$, positive control vs. CTLA4-treated and Y100Ftreated groups). 


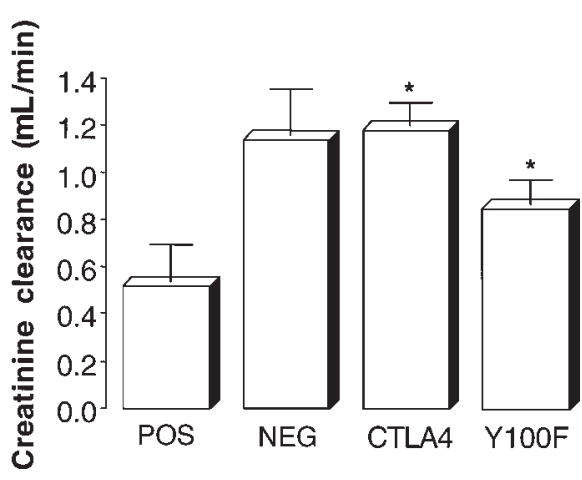

Figure 2

Effect of native CTLA4-Ig or Y100F-Ig treatment on creatinine clearance in groups of WKY rats $(n=5-8)$ with EAG. Results shown represent the mean \pm SD of each group at week 4 after immunization. ( ${ }^{*} P<0.01$, positive control vs. CTLA4-treated and Y100F-treated groups).

\section{Preparation of GBM antigen}

Collagenase-solubilized GBM was prepared from Sprague-Dawley rat kidneys as described previously $(18,21)$. Briefly, the kidneys were decapsulated, the medulla was partly removed, and the cortex was forced through sieves in order to isolate the glomeruli. After examination by light microscopy, the glomeruli were disrupted ultrasonically, and the resulting material was lyophilized and digested with purified type I collagenase for 1 hour at $37^{\circ} \mathrm{C}$.

\section{Induction of EAG}

EAG was induced in WKY rats by a single intramuscular injection of collagenase-solubilized rat GBM in an equal volume of FCA, at a dose of $5 \mathrm{mg} / \mathrm{kg}$ body weight $(18,21)$. Serial blood samples were taken by tail artery puncture under light anesthesia with isofluorane. Twenty-four-hour urine specimens were obtained by placing animals in metabolic cages.

\section{Assessment of EAG}

Rocket immunoelectrophoresis. Urinary albumin concentrations were measured in 24-hour collections from animals with EAG by rocket immunoelectrophoresis (Amersham Pharmacia Biotech, St. Albans, United Kingdom) as described previously (39). Briefly, urine samples from experimental animals were subjected to immunoelectrophoresis at $60 \mathrm{~V}$ in an electrophoresis tank containing Barbitone buffer (BDH Laboratory Supplies, Poole, United Kingdom), ( $\mathrm{pH} 9.5$ ) for 6 hours, using a $1 \%$ agarose gel (BDH Laboratory Supplies) containing rabbit antisera to rat albumin raised in our laboratory. Results were calculated using rat serum albumin standards (which were run at the same time), and were expressed in milligrams per 24 hours.

Creatinine clearance. Creatinine levels in serum and urine from animals at week 4 (the time of sacrifice) were measured by spectrometry by standard techniques. Cre- atinine clearance was then calculated for each animal and expressed as milliliters per minute.

Direct immunofluorescence. Direct immunofluorescence studies to assess deposits of IgG and fibrin within the glomeruli were carried out on kidneys obtained at sacrifice by a method similar to that previously described (40). Tissue was embedded in OCT II embedding medium (Miles Inc., Elkhart, Indiana, USA) on cork discs. Embedded tissues were snap frozen in isopentane (BDH Laboratory Supplies) that was precooled in liquid nitrogen, and then stored at $-70^{\circ} \mathrm{C}$. Cryostat sections were cut $4 \mu \mathrm{m}$ thick and were incubated with either FITC-labeled rabbit anti-rat IgG (Serotec Ltd., Oxford, United Kingdom) or goat antirat fibrin (Nordic Immunology, Tilburg, The Netherlands). The degree of immunostaining was assessed and graded from 0 to $3+$ by a blinded observer.

Light microscopy. Kidney tissue taken at the time of sacrifice was fixed in 10\% neutral-buffered formalin, processed, and embedded in paraffin wax for light microscopy by standard techniques. Briefly, $3-\mu \mathrm{m}$ sections were stained with hematoxylin and eosin, and periodic acid-Schiff. Fifty glomeruli per section were assessed and graded by a blinded observer as severe
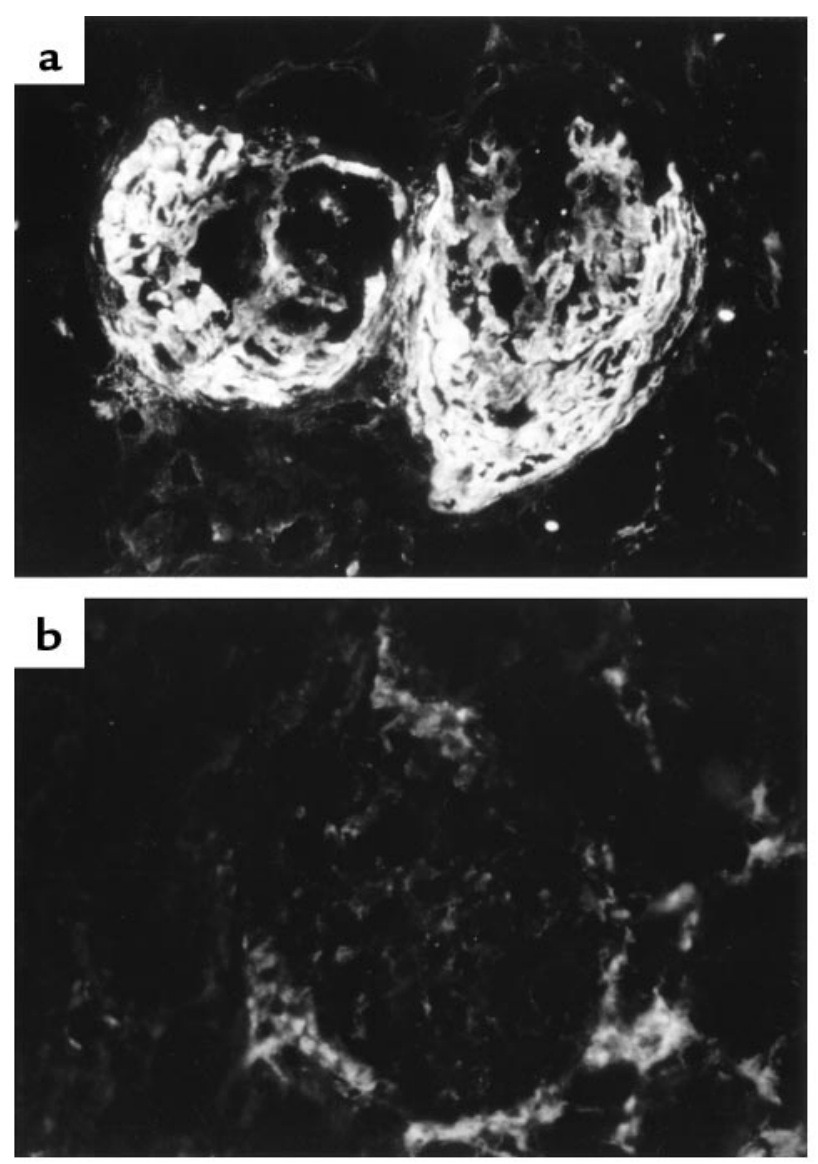

Figure 3

Direct immunofluorescence of kidney tissue at 4 weeks in WKY rats with EAG. (a) Large deposits of fibrin within the glomeruli of an animal given control fusion protein. (b) Negative findings in an animal treated with Y100F-Ig. 


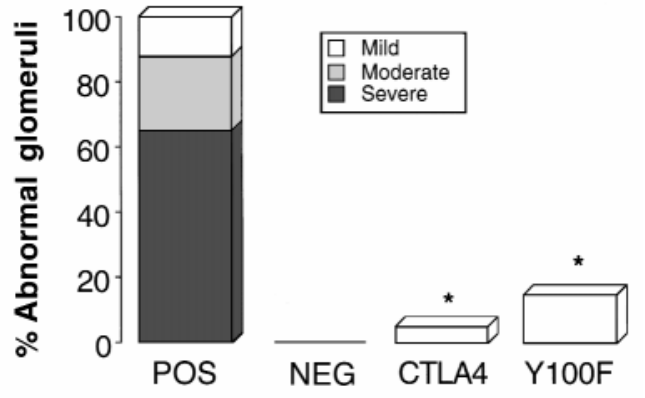

Figure 4

Effect of native CTLA4-Ig or Y100F-Ig treatment on renal histology at week 4 in groups of WKY rats $(n=5-8)$ with EAG. Results shown represent the severity of glomerular abnormalities that were graded as severe $(>50 \%$ of the glomerulus affected by necrosis or crescent formation), moderate ( $<50 \%$ of the glomerulus affected by necrosis or crescent formation), mild (small areas of glomerular abnormalities), or normal. ( $P<0.001$, positive control vs. CTLA4-treated and Y100F-treated groups).

(> $50 \%$ of the glomerulus affected by necrosis or crescent formation), moderate ( $<50 \%$ of the glomerulus affected by necrosis or crescent formation), mild (small areas of glomerular abnormalities), or normal (21).

Immunohistology. Formalin-fixed kidney sections obtained at sacrifice were stained with mAb by standard indirect immunoperoxidase staining techniques. Antibodies used were ED1 (for macrophages, at a dilution of 1:500), W3/13 (for $\mathrm{T}$ lymphocytes, at a dilution of $1: 100$ ), W3/25 (for $\mathrm{CD}^{+}$lymphocytes, at a dilution of 1:100), and OX8 (for CD8 lymphocytes, at a dilution of 1:200) (Serotec Ltd.). The cellular infiltrate was assessed by a blinded observer by counting the number of positively stained cells per 50 consecutive glomeruli.

Flow cytometry analysis of isolated glomerular cells. Purified glomeruli were dissociated into single cells by a 3 -stage digestion procedure using trypsin, DNAse, and collagenase, as described previously (41). At the end of the digestion, cells were assessed for viability using fluorescein diacetate and ethidium bromide. Isolated glomerular cells were then labeled with mAbs OX1 (for leukocytes), W3/13 (for T lymphocytes), W3/25 (for $\mathrm{CD}^{+}$lymphocytes), OX8 (for CD8 ${ }^{+}$lymphocytes), and OX23 (antibody to human factor $\mathrm{H}$ ) (as an irrelevant isotype-matched control). All antibodies were used at a dilution of 1:100. After antibody staining, the cells were fixed in $2 \%$ paraformaldehyde and were quantified by flow cytometry analysis (Coulter EPICS XL, Luton, United Kingdom). The single-cell suspension obtained after digestion contained a mixed population of cells, so the flow cytometry analyzer was programmed to exclude debris and fragments too small to be cells. Immediately before analysis, propidium iodide was added to identify the cells that were gated for assessment. Stained cells were expressed as a percentage of total leukocytes, after subtraction of the irrelevant isotype-matched control (OX23).

Circulating anti-GBM antibody concentrations. Circulating anti-GBM antibodies were measured in sera from ani- mals with EAG by solid-phase ELISA in a procedure similar to that previously described (21). Microtiter plates (GIBCO BRL, Paisley, United Kingdom) were coated with collagenase-digested rat GBM by overnight incubation at $4^{\circ} \mathrm{C}$, and test or control sera were applied for 1 hour at $37^{\circ} \mathrm{C}$. Bound anti-GBM antibody was detected by horseradish peroxidase-conjugated sheep anti-rat IgG (SigmaAldrich Company Ltd., Poole, United Kingdom), and was developed using the substrate $o$-phenylenediamine dihydrochloride (Sigma-Aldrich Company Ltd.). The absorbance for each well was read at $492 \mathrm{~nm}$ using an Anthos Multiskan ELISA plate reader (Labtech International, Uckfield, United Kingdom), and the results were initially expressed as the mean optical density of each triplicate sample. To compare different experimental groups, results were finally expressed as a percentage of the binding obtained with a positive reference serum (23). To check the specificity of these antibodies, a similar ELISA was used, but plates were coated with human recombinant $\alpha 3$ (IV)NC1, as described previously $(42,43)$.

Isotype analysis of anti-GBM antibodies. Circulating levels of IgG1 and IgG2a anti-GBM antibodies were measured in sera from animals with EAG at week 4 after immunization, by an indirect ELISA similar to that previously described (44). Briefly, microtiter plates were coated with collagenase-digested rat GBM or human recombinant $\alpha 3(\mathrm{IV}) \mathrm{NC} 1$, and test or control sera was applied at dilutions of $1: 30,1: 100,1: 300$, and 1:1,000. The isotypes of circulating anti-GBM antibodies were detected by mouse $\mathrm{mAb}$ specific for rat IgG1 and IgG2a, followed by goat anti-mouse IgG (Serotec Ltd.). Levels of bound IgG1 and IgG2a anti-GBM antibodies were detected by alkaline phosphatase-conjugated rabbit anti-goat IgG (Sigma-Aldrich Company Ltd.), and were developed using the substrate $p$-nitrophenyl phosphate (SigmaAldrich Company Ltd.). The absorbance for each well was read at $405 \mathrm{~nm}$, and the results were expressed as the mean optical density of each triplicate sample.

\section{Experimental protocol}

Groups of animals $(n=5-8)$ immunized with rat GBM in FCA were given either murine CTLA4 fused with murine IgG, mutant murine CTLA4 (Y100F) (which selectively

\section{Table 1}

Summary of results from WKY rats with EAG

\begin{tabular}{|c|c|c|c|c|}
\hline \multirow[t]{2}{*}{ Group } & \multirow{2}{*}{$\begin{array}{l}\text { Anti-GBM Ab } \\
\text { (\% binding) }\end{array}$} & \multirow{2}{*}{$\begin{array}{c}\text { Albuminuria } \\
\text { (mg/day) }\end{array}$} & \multicolumn{2}{|c|}{$\begin{array}{c}\text { Abnormal } \\
\text { glomeruli (\%) }\end{array}$} \\
\hline & & & Total & severe \\
\hline EAG (L6) $(n=5)$ & $110 \pm 6$ & $485 \pm 75$ & $100 \pm 0$ & $60 \pm 25$ \\
\hline EAG (saline) $(n=8)$ & $100 \pm 14$ & $509 \pm 91$ & $100 \pm 0$ & $65 \pm 15$ \\
\hline FCA (saline) $(n=5)$ & $20 \pm 2$ & 0 & 0 & 0 \\
\hline $1-\mathrm{mlg}(n=7)$ & $45 \pm$ & $15 \pm 8$ & $5 \pm 3$ & 0 \\
\hline hCTLA4-hlg $(n=8)$ & $72 \pm 8$ & $35 \pm 14$ & $15 \pm 8$ & 0 \\
\hline $\mathrm{mY100F-mlg}(n=5)$ & $90 \pm 10$ & $30 \pm 12$ & $15 \pm 12$ & 0 \\
\hline hY100F-hlg $(n=6)$ & $105 \pm 12$ & $50 \pm 21$ & $20 \pm 7$ & 0 \\
\hline
\end{tabular}

WKY rats with EAG were treated with murine $(\mathrm{m})$ CTLA4 or $\mathrm{Y} 100 \mathrm{~F}$ fused with $\mathrm{mlgG}$, and human (h) CTLA4 or Y100F fused with human IgG. L6 is a control fusion protein. Results are expressed as the mean \pm SD of each group at week 4 after immunization. 

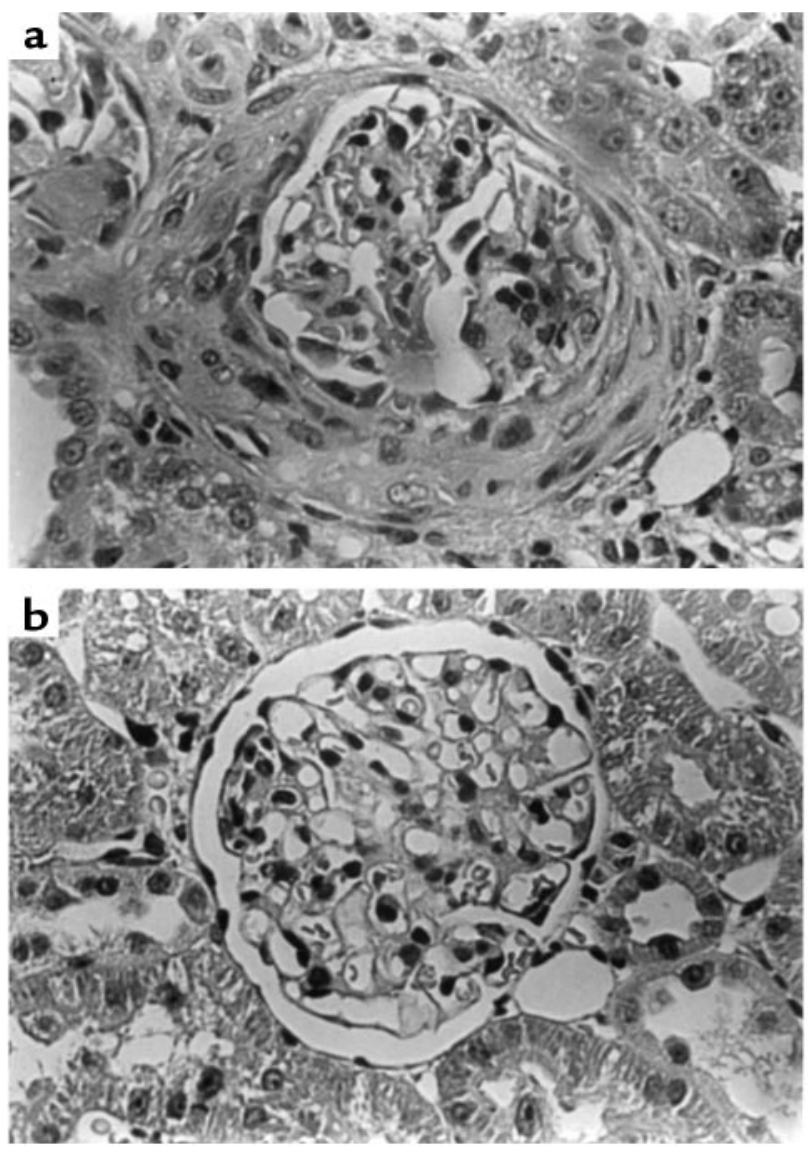

Figure 5

Light microscopy of kidney tissue at 4 weeks in WKY rats with EAG. (a) Marked segmental necrosis of the glomerular tuft with crescent formation in an animal given control fusion protein. (b) A mildly affected glomerulus in an animal treated with Y100F-Ig.

blocks B7.1) fused with murine IgG, human CTLA4 fused with human IgG, or mutant human CTLA4 (Y100F) fused with human IgG. All treated groups of animals were given the fusion proteins intravenously at a dose of $1 \mathrm{mg}$ at the time of immunization, and then $0.1 \mathrm{mg}$ intraperitoneally 3 times per week for the duration of the study (4 weeks). Positive-control groups given rat GBM in FCA were given a control fusion protein (specific for the L6 tumor-associated antigen) or saline, and a negative-control group (FCA alone) was given saline.

\section{Statistical analysis}

Differences between data were determined by MannWhitney $U$ test. ANOVA was used to confirm differences between multiple data sets.

\section{Results}

Results from our initial pilot studies (37) showed that continued treatment with human CTLA4 fused with human $\mathrm{IgG}$ at a dose of $1 \mathrm{mg}$ intravenously at the time of immunization, and then $0.1 \mathrm{mg}$ intraperitoneally 3 times per week for 4 weeks (the duration of the study), was effective in reducing the severity of EAG in the WKY rat. In these studies we also demonstrated that a single high dose at the time of immunization was less effective than continued treatment.

In this study, we examined the effects of continued in vivo administration of murine and human CTLA4, fused respectively with murine or human IgG. In addition, we examined the effects of a mutant form of murine and human CTLA4 (Y100F), which selectively blocks B7.1, fused respectively with murine or human IgG. In both cases, the murine construct was more effective in the treatment of EAG than was the human construct (results are summarized in Table 1). As a result of this observation, subsequent results reported in this paper are from groups of animals treated with native murine CTLA4 or Y100F fused with murine IgG.

Albuminuria. All positive-control rats immunized with rat GBM in FCA produced detectable levels of albumin in the urine by week 2 , which increased by week 3 and peaked at week 4. Animals given native CTLA4-Ig or Y100F-Ig showed a significant reduction in albumin excretion, to nearly undetectable levels, at all time points. Negative-control animals given FCA alone did not develop albuminuria (Figure 1).

Creatinine clearance. All negative-control animals given FCA alone showed normal creatinine clearance, in the range of $1.2-1.5 \mathrm{~mL} / \mathrm{min}$. Positive control rats immunized with rat GBM in FCA showed a significant reduction in creatinine clearance. Animals treated with native CTLA4-Ig had a creatinine clearance similar to that of negative-control animals given FCA alone, whereas those given Y100F-Ig showed a slight (not significant) reduction in creatinine clearance (Figure 2).

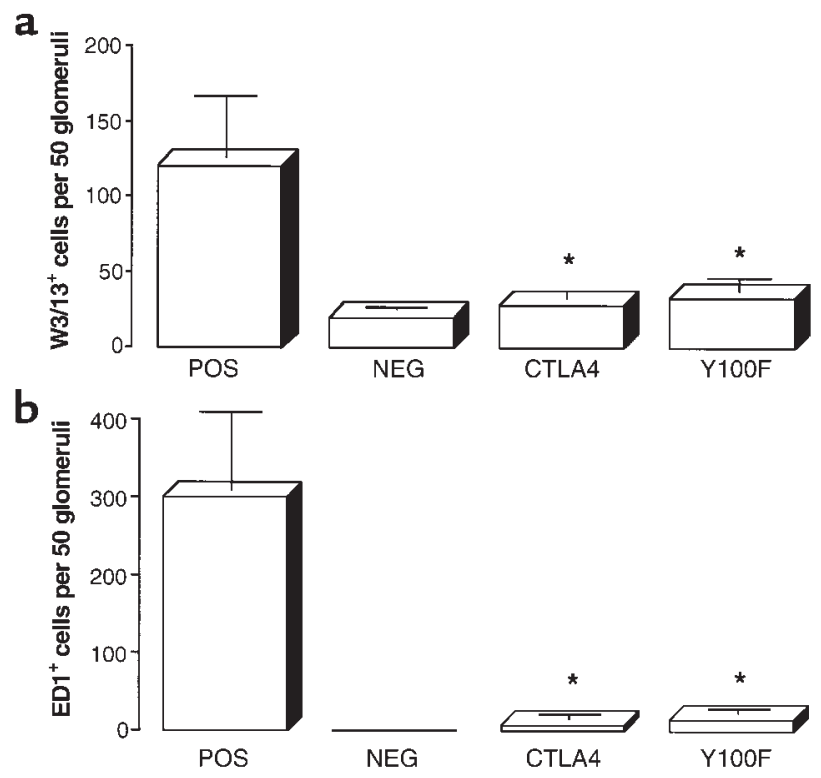

\section{Figure 6}

Effect of native CTLA4-Ig or Y100F-Ig treatment on the numbers of (a) T cells and (b) macrophages, per 50 glomerular sections, detected by immunohistology of kidney tissue in groups of WKY rats $(n=$ 5-8) with EAG. Results shown represent the mean \pm SD of each group at week 4 after immunization. ( ${ }^{*} P<0.001$, positive control vs. CTLA4-treated and Y100F-treated groups). 


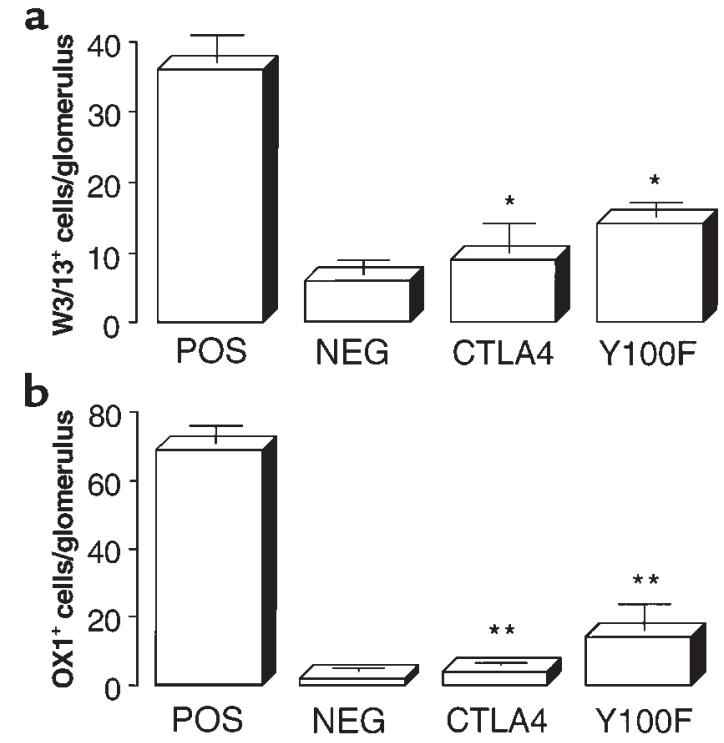

Figure 7

Flow cytometry analysis of isolated glomerular cells from groups of WKY rats treated with native CTLA4-Ig or Y100F-Ig, showing numbers of (a) $T$ cells and $(\mathbf{b})$ leukocytes, per glomerulus. Results shown represent the mean \pm SD of each group at week 4 after immunization. ( ${ }^{*} P<0.01 ;{ }^{*} P$ $<0.005$, positive control vs. CTLA4-treated and Y100F-treated groups).

Direct immunofluorescence for fibrin. Direct immunofluorescence for fibrin on kidney tissue at 4 weeks revealed that positive-control animals given rat GBM in FCA showed large fibrin deposits within the glomeruli. Animals treated with native CTLA4-Ig or Y100F-Ig had no detectable deposits of fibrin within the glomeruli. Negative-control animals given FCA alone also showed no binding. Results are summarized in Table 2 and are illustrated in Figure 3.

Light microscopy. Light microscopy of kidney tissue at week 4 revealed that positive-control animals given rat GBM in FCA showed severe focal necrotizing glomerulonephritis with crescent formation. Animals treated with native CTLA4-Ig or Y100F-Ig showed no histological evidence of fibrinoid necrosis or crescent formation. Only mild glomerular abnormalities were observed, in a minority of the glomeruli examined. negative-control animals given FCA alone showed normal renal histology. Results are shown in Figure 4 and are illustrated in Figure 5.

Analysis of infiltrating glomerular cells. Immunohistology and flow cytometry analysis of isolated glomerular cells at week 4 showed that positive-control animals given rat GBM in FCA had a glomerular infiltrate of $\mathrm{T}$ lymphocytes (which were predominantly $\mathrm{CD}^{+}$) and macrophages. Animals treated with native CTLA4-Ig or Y100F-Ig showed a marked reduction in infiltration of both $\mathrm{T}$ cells and macrophages, similar to levels in negative-control animals given FCA alone. Results of immunohistology analysis are shown in Figure 6; results of flow cytometry analysis are shown in Figure 7.
Circulating anti-GBM antibody concentrations. All positive-control rats immunized with rat GBM in FCA, and given either saline or control fusion protein, produced detectable levels of circulating anti-GBM antibody by week 2 , which increased to high levels by week 3 and peaked at week 4. Animals given native CTLA4-Ig showed a significant reduction in circulating anti-GBM antibody concentrations at all time points, similar to levels in negative-control animals given FCA alone. By contrast, animals given Y100F-Ig showed no significant reduction in total circulating anti-GBM antibodies (Figure 8). Similar results were obtained using recombinant human $\alpha 3(\mathrm{IV}) \mathrm{NC} 1$ as the ligand (not shown).

Circulating anti-GBM antibody isotypes. All positive-control rats immunized with rat GBM in FCA produced detectable levels of IgG1 and IgG2a anti-GBM antibodies by week 4. Animals given native CTLA4-Ig showed a significant reduction in the levels of both IgG1 and IgG2a anti-GBM antibodies, similar to levels in negative-control animals given FCA alone. By contrast, animals given Y100F-Ig showed a significant reduction in IgG2a antibodies, but not IgG1 antibodies (Figure 9). Similar results were obtained using recombinant human $\alpha 3$ (IV)NC1 as the ligand (not shown).

Direct immunofluorescence measurement of IgG. Direct immunofluorescence measurement of IgG in kidney tissue at 4 weeks revealed that positive-control animals given rat GBM in FCA showed strong linear deposits of IgG along the GBM, and to a lesser extent along the tubular basement membrane. Animals treated with native CTLA4-Ig showed a marked reduction in deposits of IgG on the GBM, whereas those given Y100F-Ig demonstrated only a moderate reduction in these deposits. Negative-control animals given FCA alone showed no antibody binding. Results are summarized in Table 2 and are illustrated in Figure 10.

\section{Discussion}

Although T-cell costimulation caused by interactions between CD28 and B7 appears to be required for the

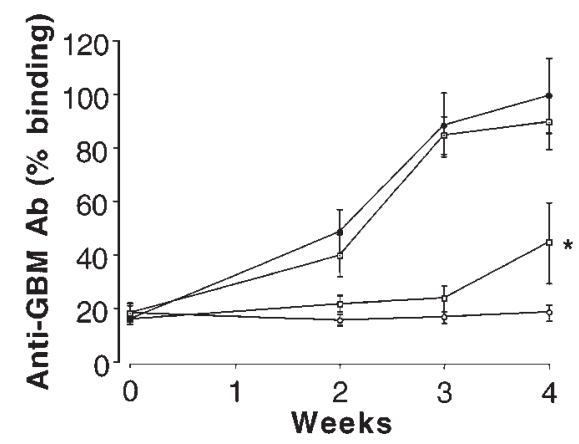

Figure 8

Effect of native CTLA4-Ig or Y100F-Ig treatment on circulating anti-GBM antibody concentrations in groups of WKY rats $(n=5-8)$ with EAG. Results shown represent the mean \pm SD of each group: positive control (filled circles), CTLA4-Ig (filled squares), Y100F-Ig (open squares), and negative control (open circles). ( ${ }^{*} P<0.001$; positive control vs. CTLA4-treated group). 


\section{a}

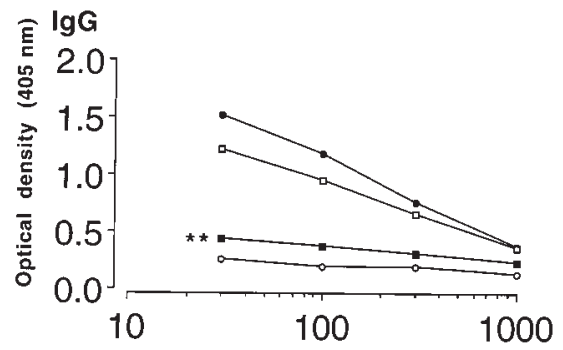

b

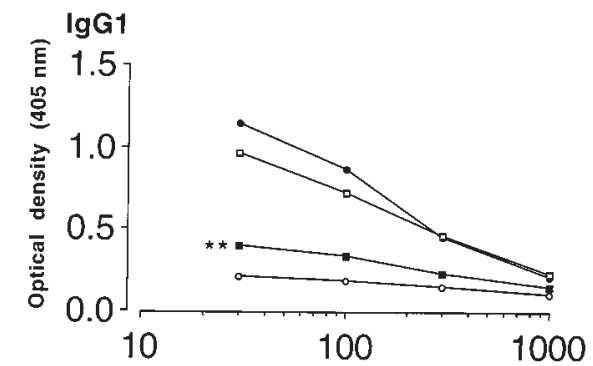

C

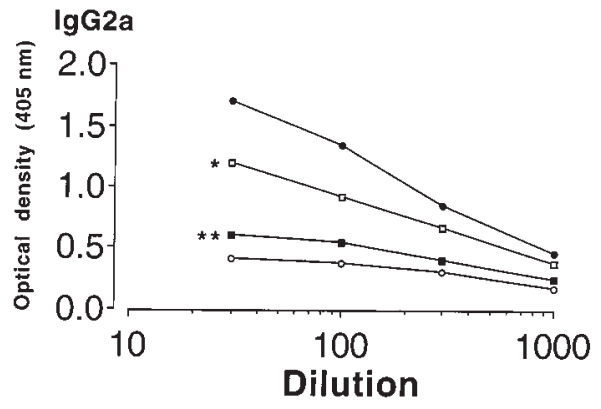

Figure 9

Effect of native CTLA4-Ig or Y100F-Ig treatment on levels of circulating (a) $\lg G(\mathbf{b}) \lg G 1$, and (c) $\lg G 2 a$ anti-GBM antibodies in groups of WKY rats $(n=5-8)$ with EAG. Results shown represent the mean of each group at week 4 after immunization: positive control (filled circles), CTLA4-Ig (filled squares), Y100F-Ig (open squares), and negative control (open circles). ( ${ }^{*} P<0.01 ;{ }^{*} P<0.001$, positive control vs. CTLA4-treated and Y100F-treated groups).

development of many experimental autoimmune diseases, the respective roles of $\mathrm{B} 7.1$ and $\mathrm{B} 7.2$ remain unclear. To address this issue, we have examined the effect of CD28-B7 blockade, and of selective blockade of B7.1, on the development of EAG in the WKY rat. Previous work in rat models of autoimmunity, although limited, has demonstrated that CD28-B7 costimulatory blockade by CTLA4-Ig is effective in inhibiting disease $(36,45,46)$. In a similar model of EAG in the WKY rat, induced by immunization with bovine GBM, administration of human CTLA4-Ig reduced the severity of disease, even when injections were begun after the onset of glomerulonephritis (36). In experimental autoimmune encephalomyelitis in the Lewis rat, it has been shown that blockade of CD28-B7 by systemic administration of CTLA4-Ig was effective in preventing and treating induced disease (45). Similarly, in collagen type II-induced arthritis (CIA), administration of CTLA4-Ig from the time of immunization prevented the development of CIA, whereas treatment during ongoing disease resulted in amelioration of arthritis (46). As in the mouse, there are conflicting data as to the respective roles of B7.1 and B7.2 ligands in the autoimmune response in the rat. In CIA, mAbs against B7.1 or B7.2 had no effect on the course of disease when given alone, but resulted in reduced severity of disease when given together (46). Therefore, it remains unclear whether, in the rat, B7.1 costimulation is mainly responsible for a Th1-like response, and B7.2 for a Th2-like response.

In this study, CTLA4-Ig given at a dose of $1 \mathrm{mg}$ intravenously at the time of immunization, and then 0.1 mg intraperitoneally 3 times per week for the duration of the study, resulted in a marked reduction in circulating and deposited anti-GBM antibodies, albuminuria, deposits of fibrin in the glomeruli, severity of glomerular abnormalities, and numbers of infiltrating $\mathrm{T}$ cells and macrophages. These results are consistent with those of Nishikawa et al. in a similar model (36), but show a greater effect of CTLA4-Ig. However, in their study, animals developed antibody to the human CTLA4-Ig, which resulted in a reduction in circulating CTLA4-Ig levels. In our study, using mouse CTLA4 conjugated to mouse Ig, the increased effectiveness in treating the disease may be due to the fact that mouse
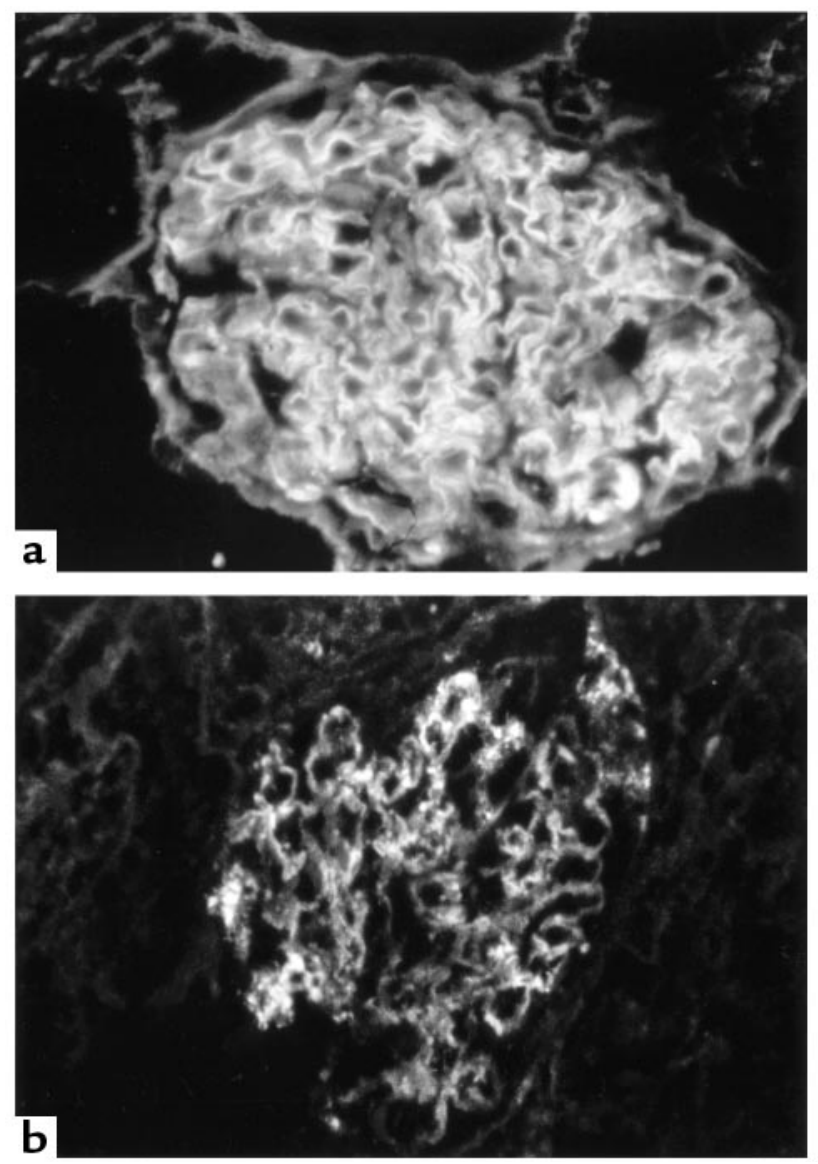

Figure 10

Direct immunofluorescence of kidney tissue at 4 weeks in WKY rats with EAG. (a) Strong linear deposition of IgG along the GBM in an animal given control fusion protein. (b) Marked reduction is seen in the deposition of IgG on the GBM in an animal treated with native CTLA4-Ig. 


\section{Table 2}

Effects of native CTLA4-Ig or Y100F-Ig treatment in WKY rats with EAG on deposition of fibrin in the glomeruli and deposition of IgG on the GBM

\section{Fibrin}

$\begin{array}{lcccc} & 3+ & 2+ & 1+ & - \\ \text { Positive } & 5 & 3 & 0 & 0 \\ \text { Negative } & 0 & 0 & 0 & 5 \\ \text { CTLA4-Ig } & 0 & 0 & 0 & 7 \\ \text { Y100F-Ig } & 0 & 0 & 0 & 5\end{array}$

IgG

$\begin{array}{lcccc} & 3+ & 2+ & 1+ & - \\ \text { Positive } & 6 & 2 & 0 & 0 \\ \text { Negative } & 0 & 0 & 0 & 5 \\ \text { CTL4-Ig } & 0 & 0 & 2 & 5 \\ \text { Y100F-Ig } & 0 & 2 & 3 & 0\end{array}$

Results are expressed as intensity of fluorescence at week 4 in individual animals.

Ig is less immunogenic in the rat than is human Ig. Our results clearly demonstrate that blockade of the CD28-B7 costimulatory pathway completely prevents the development of crescents in this model of EAG. This is probably due to inhibition of T-cell activation resulting in a reduction of both autoantibody production and the generation of effector $T$ cells, and suggests that CTLA4-Ig successfully blocked both B7.1 and B7.2 on APC.

Interestingly, treatment with Y100F-Ig, which binds only to B7.1, also resulted in a reduction in the severity of nephritis, but produced no significant reduction in overall circulating anti-GBM antibodies. However, Y100F-Ig did produce a moderate reduction in Th1-dependent IgG2a anti-GBM antibodies. It also prevented fibrin deposition and infiltration by $T$ cells and macrophages - features that are suggestive of a Th1-like response in the glomeruli. This observation is consistent with that in an animal model of airway eosinophilia, in which blocking B7.1 with Y100F-Ig prevented antigen-induced accumulation of eosinophils and lymphocytes in the lung, but did not block systemic blood eosinophilia or IgE antibody production (10). Our results could be interpreted in 2 ways. First, it could be that interactions between T cells and APC in the rat are more B7.1-dependent than are T cell-B cell interactions, because the $\mathrm{T}$-cell response appeared to be modulated by either CTLA4-Ig or Y100FIg. This suggests that B7.1 is the dominant ligand for T cells, which provide help for effector $\mathrm{T}$ cells, and that B7.2 is the more important ligand in providing T-cell help for $\mathrm{B}$ cells. This would be consistent with the hypothesis that B7.1 and B7.2 are the preferential ligands in stimulating a Th1- or Th2-like response, respectively, and is supported by the observation that B7.1 blockade reduced IgG2a antibody levels. Alternatively, our results may indicate that a lower level of overall T-cell activation (which could be provided by $\mathrm{CD} 28$ interaction with $\mathrm{B} 7.2$ alone) is required to induce a $\mathrm{B}$-cell response than to cause direct cell-mediated renal injury. However, the switching of Ig isotype classes in the Y100F-Ig-treated group suggests a more complex explanation. These observations may have relevance for the treatment of autoimmune disease, because therapeutic strategies aimed at B7.1 could provide beneficial effects for autoimmune responses, while allowing normal responses to certain pathogens through CD28-B7.2 interactions.

In conclusion, we have demonstrated that blockade of CD28-B7 with fusion protein CTLA4-Ig prevented all aspects of the immune response and the development of crescentic nephritis in EAG. Selective blockade of B7.1 was almost as effective in preventing glomerular injury, but appeared to reduce only Th1-dependent immune responses. The simplest explanation for these findings is that EAG in the WKY rat is a predominantly Th1-dependent disease, and that B7.1 preferentially stimulates a Th1-like response. However, further work is clearly required, including analysis of cytokine profiles from autoreactive T cells in EAG. The clinical relevance of this study is that strategies selectively targeting T-cell costimulation may provide a novel approach to the treatment of Goodpasture's disease.

\section{Acknowledgments}

F.W.K. Tam is supported by a National Kidney Research Fund Senior Fellowship. A. Chandraker is the recipient of a Juvenile Diabetes Foundation International Fellowship Grant. M.H. Sayegh is the recipient of a National Kidney Foundation Clinical Scientist Award. We are grateful to Bristol-Myers Squibb Co. for kindly supplying the CTLA4, Y100F, and L6 fusion proteins, and to the National Kidney Research Fund, United Kingdom, for supporting this study.

1. Mueller, D.L.,Jenkins, M.K., and Schwartz, R.H. 1989. Clonal expansion versus functional clonal inactivation: a costimulation signalling pathway determines the outcome of T cell antigen receptor occupancy. Annu. Rev. Immunol. 7:445-480.

2. June, C.H., Ledbetter, J.A., Linsley, P.S., and Thompson, C.B. 1990. Role of CD28 receptor in T cell activation. Immunol. Today. 11:211-216.

3. Linsley, P.S., et al. 1991. CTLA-4 is a second receptor for the B cell activation antigen B7. J. Exp. Med. 174:561-569.

4. Linsley, P.S., et al. 1992. Coexpression and functional cooperation of CTLA4 and CD28 on activated T lymphocytes. J. Exp. Med. 176:1595-1604.

5. Lane, P., Gerhard, W., Hubele, S., Lanzavecchia, A., and McConnell, J. 1993. Expression and functional properties of mouse B7/BB1 using a fusion protein between mouse CTLA-4 and human IgG1. Immunology. 80:56-61.

6. Linsley, P.S., and Ledbetter,J.A.1993. The role of CD28 receptor during T cell responses to antigen. Annu. Rev. Immunol. 11:191-212.

7. Bluestone, J.A. 1995. New perspectives of CD28-B7 mediated T cell costimulation. Immunity. 2:555-559.

8. Thompson, C.B. 1995. Distinct roles for the costimulatory ligands B7-1 and B7-2 in Thelper cell differentiation. Cell. 81:979-982.

9. Sayegh, M.H., et al. 1995. CD28-B7 blockade after alloantigenic challenge in vivo inhibits Th1 cytokines but spares Th2. J. Exp. Med. 181:1869-1874.

10. Harris, N., et al. 1997. CD80 costimulation is essential for the induction of airway eosinophilia. J. Exp. Med. 185:177-182.

11. Sayegh, M.H., and Turka, L.A. 1998. The role of T-cell costimulatory activation pathways in transplant rejection. N. Engl. J. Med. 338:1813-1821.

12. Finck, B.K., Linsley, P.S., and Wofsy, D. 1994. Treatment of murine lupus with CTLA4Ig. Science. 265:1225-1227.

13. Cross, A.H., et al. 1995. Long-term inhibition of murine experimental allergic encephalomyelitis. Using CTLA4-Fc supports a key role for CD28 costimulation. J. Clin. Invest. 95:2783-2789.

14. Khoury, S.J., et al. 1995. CD28-B7 costimulatory blockade by CTLA4Ig prevents actively induced experimental autoimmune encephalomyelitis and inhibits Th1 but spares Th2 cytokines in the central nervous system. J. Immunol. 155:4521-4524.

15. Lenschow, D.J., et al. 1995. Differential effects of anti-B7-1 and anti-B7-2 monoclonal antibody treatment on the development of diabetes in the 
nonobese diabetic mouse. J. Exp. Med. 181:1145-1155.

16. Perrin, P.J., et al. 1995. Role of B7:CD28/CTLA-4 in the induction of chronic relapsing experimental allergic encephalomyelitis J. Immunol. 154:1481-1490.

17. Akalin, E., Chandraker, A., Sayegh, M.H., and Turka, L.A. 1997. Role of CD28-B7 costimulatory interaction in alloimmune responses. Kidney Int. 58(Suppl.):S8-S10.

18. Pusey, C.D., et al. 1991. Experimental autoimmune glomerulonephritis induced by homologous and isologous glomerular basement membrane in Brown-Norway rats. Nephrol. Dial. Transplant. 6:457-465.

19. Sado, Y., and Naito, I. 1987. Experimental autoimmune glomerulonephritis induced in rats by soluble isologous or homologous antigens from glomerular and tubular basement membranes. Br.J. Exp. Pathol. 68:695-704.

20. Bolton, W.K., May, W.J., and Sturgill, B.C. 1993. Proliferative autoimmune glomerulonephritis in rats: a model for autoimmune glomerulonephritis in humans. Kidney Int. 44:294-306.

21. Reynolds, J., Mavronmatidis, K., Cashman, S.J., Evans, D.J., and Pusey, C.D. 1998. Experimental autoimmune glomerulonephritis (EAG) induced by homologous and heterologous glomerular basement membrane in two substrains of Wistar Kyoto rat. Nephrol. Dial. Transplant. 13:44-52.

22. Reynolds, J., Syrganis, C., Derry, C.J., and Pusey, C.D. 1994. Comparison of the specificity of anti-GBM antibodies in EAG and Goodpasture's syndrome. Nephrol. Dial. Transplant. 9:849. (Abstr.)

23. Sado, Y., Kagawa, M., Naito, I., and Okigaki, T. 1991. Properties of bovine nephritogenic antigen that induces anti-GBM nephritis in rats and its similarity to the Goodpasture antigen. Virchows Arch. B Cell Pathol. Incl. Mol. Pathol. 60:345-351.

24. Sado, Y., et al. 1997. Purification and characterization of human nephritogenic antigen that induces anti-GBM nephritis in rats. J. Pathol. 182:225-232.

25. Sado, Y., et al. 1998. Induction of anti-GBM nephritis in rats by recombinant $\alpha 3(\mathrm{IV}) \mathrm{NC} 1$ and $\alpha 4(\mathrm{IV}) \mathrm{NC} 1$ of type IV collagen. Kidney Int. 53:664-671.

26. Abbate, M., et al. 1998. Experimental Goodpasture's syndrome in WistarKyoto rats immunized with $\alpha 3$ chain of type IV collagen. Kidney Int. 54:1550-1561.

27. Sado, Y., Naito, I., and Okigaki, T. 1989. Transfer of anti-glomerular basement membrane antibody-induced glomerulonephritis in inbred rats with isologous antibodies from urine of nephritic rats. J. Pathol. 158:325-332.

28. Kalluri, R., Danoff, T.M., Okada, H., and Neilson, E.G. 1997. Susceptibility to anti-glomerular basement membrane disease and Goodpasture's syndrome is linked to MHC class II genes and the emergence of T cell-mediated immunity in mice. J. Clin. Invest. 100:2263-2275.

29. Reynolds, J., et al. 1998. Ultrastructural abnormalities of kidney and lung in an animal model of Goodpasture's disease. J. Am. Soc. Nephrol. 9:466. (Abstr.)

30. Reynolds, J., Sallie, B.A., Syrganis, C., and Pusey, C.D. 1993. The role of Thelper lymphocytes in priming for experimental autoimmune glomeru- lonephritis in the BN rat. J. Autoimmun. 6:571-585.

31. Reynolds, J., and Pusey, C.D. 1995. T cell responses to autoantigenic components of GBM in EAG. J. Am. Soc. Nephrol. 6:850. (Abstr.)

32. Reynolds, J., Cashman, S.J., Evans, D.J., and Pusey, C.D. 1991. Cyclosporin A in the prevention and treatment of experimental autoimmune glomerulonephritis in the Brown Norway rat. Clin. Exp. Immunol. 85:28-32.

33. Reynolds, J., and Pusey, C.D. 1994. In vivo treatment with a monoclonal antibody to $T$ helper cells in experimental autoimmune glomerulonephritis. Clin. Exp. Immunol. 95:122-127.

34. Reynolds, J., Norgan, V.A., Chana, U., and Pusey, C.D. 1999. Anti-CD8 monoclonal antibody therapy is effective in experimental autoimmune glomerulonephritis. J. Am. Soc. Nephrol. 10:519. (Abstr.)

35. Reynolds, J., Newman, V.O., and Pusey, C.D. 1996. Oral administration of GBM to WKY rats prevents the development of EAG. J. Am. Soc. Nephrol. 7:1717. (Abstr.)

36. Nishikawa, K., et al. 1994. Effect of CTLA-4 chimeric protein on rat autoimmune anti-glomerular basement membrane disease. Eur. J. Immunol. 24:1249-1254.

37. Reynolds, J., et al. 1997. Effect of fusion protein CTLA4-Ig on the development of experimental autoimmune glomerulonephritis (EAG) in the WKY rat. Kidney Int. 52:117. (Abstr.)

38. Faquim-Mauro, E.L., Coffman, R.L., Abrahamsohn, I.A., and Macedo, M.S. 1999. Mouse IgG1 antibodies comprise two functionally distinct types that are differentially regulated by IL-4 and IL-12. J. Immunol. 163:3572-3576.

39. Laurell, C.B. 1966. Quantitative estimation of proteins by electrophoresis in agarose gel containing antibodies. Anal. Biochem. 15:45-52.

40. Cashman, S.J., Pusey, C.D., and Evans, D.J. 1988. Extraglomerular distribution of immunoreactive Goodpasture antigen. J. Pathol. 155:61-70.

41. Cook, H.T., Smith, J., and Cattell, V. 1987. Isolation and characterization of inflammatory leukocytes from glomeruli in an in situ model of glomerulonephritis in rats. Am. J. Pathol. 126:126-136.

42. Turner, N., et al. 1992. Molecular cloning of the human Goodpasture antigen demonstrates it to be the $\alpha 3$ chain of type IV collagen. J. Clin. Invest. 89:592-601.

43. Turner, N., Forstova, J., Rees, A., Pusey, C.D., and Mason, P.J. 1994. Production and characterization of recombinant Goodpasture antigen in insect cells. J. Biol. Chem. 269:17141-17145.

44. Cohen, S.B., and Weetman, A.P. 1990. Serological analysis of experimental autoimmune thyroiditis in the buffalo strain rat. Int. Arch. Allergy Appl. Immunol. 91:47-53.

45. Gallon, L., et al. 1997. Differential effects of B7-1 blockade in the rat experimental autoimmune encephalomyelitis model. J. Immunol. 159:4212-4216.

46. Webb, L.M.C., Walmsley, M.J., and Feldmann, M. 1996. Prevention and amelioration of collagen-induced arthritis by blockade of the CD28 co-stimulatory pathway: requirement for both B7-1 and B7-2. Eur. J. Immunol. 26:2320-2328. 